\title{
Differential Effects of Recombinant Adeno-Associated Virus-Mediated Neuropeptide Y Overexpression in the Hypothalamic Paraventricular Nucleus and Lateral Hypothalamus on Feeding Behavior
}

\author{
Birgitte Tiesjema, ${ }^{1}$ Roger A. H. Adan, ${ }^{1}$ Mieneke C. M. Luijendijk, ${ }^{1}$ Andries Kalsbeek, ${ }^{2}$ and Susanne E. la Fleur ${ }^{1}$ \\ ${ }^{1}$ Rudolf Magnus Institute of Neuroscience, Department of Pharmacology and Anatomy, University Medical Center Utrecht, 3584 CG Utrecht, The \\ Netherlands, and ${ }^{2}$ Netherlands Institute for Neuroscience, 1105 AZ Amsterdam, The Netherlands
}

It is well known that neuropeptide Y (NPY) increases food intake. The hypothalamic paraventricular nucleus (PVN) and the lateral hypothalamus (LH) are both involved in the acute, hyperphagic effects of NPY. Although it is obvious that increased energy intake may lead to obesity, it is less understood which aspects of feeding behavior are affected and whether one or multiple neural sites mediate the effects of long-term increased NPY signaling. By long-term overexpressing NPY in either the PVN or the LH, we uncovered brain site-specific effects of NPY on meal frequency, meal size, and diurnal feeding patterns. In rats injected with adeno-associated virus-NPY in the PVN, increased food intake resulted from an increase in the amount of meals consumed, whereas in rats injected in the LH, increased food intake was attributable to increased meal size. Interestingly, food intake and body weight gain were only temporarily increased in PVN-injected rats, whereas in LH-injected rats hyperphagia and body weight gain remained for the entire $50 \mathrm{~d}$. Moreover, in LH-NPY rats, but not in PVN-NPY rats, diurnal rhythmicity with regard to food intake and body core temperature was lost. These data clearly show that the NPY system differentially regulates energy intake and energy expenditure in the PVN and LH, which together adjust energy balance.

Key words: rAAV; obesity; meal frequency; meal size; rhythmicity; lateral hypothalamus

\section{Introduction}

Abundant caloric intake is one of the main contributors to the current obesity epidemic. Neuropeptide Y (NPY) is one of many neuropeptides involved in feeding regulation. Although most neuropeptides exert their effects on caloric intake via increased meal size (Williams et al., 2002; Kowalski et al., 2004; Zheng et al., 2005), NPY affects both meal size (Marin Bivens et al., 1998; Baird et al., 2006) and meal frequency (Seeley et al., 1995; Ammar et al., 2000; Sederholm et al., 2002; Benoit et al., 2005). Many of these results are obtained from central NPY infusions. Given the multiple sites of action, it is likely that NPY has different functions at different brain sites. Other investigators have used intranuclear injections of NPY to study this and showed that the perifornical area/lateral hypothalamus (LH) is more sensitive to an acute injection of NPY and affects meal size (Marin Bivens et al., 1998), whereas in the paraventricular nucleus of the hypothalamus (PVN), a $4 \mathrm{~h}$ infusion of NPY affects meal frequency (Kalra et al., 1988). However, this approach is limited to the relatively

\footnotetext{
Received July 19, 2007; revised Sept. 26, 2007; accepted Nov. 11, 2007.

This study was supported by Netherlands Organization for Scientific Research ZonMW 903-039-193. H. Spierenburg is gratefully acknowledged for assistance with the radioimmunoassays.

Correspondence should be addressed to Dr. Roger A. H. Adan, Rudolf Magnus Institute of Neuroscience, Universiteitsweg 100, 3584 CG Utrecht, The Netherlands. E-mail: r.a.h.adan@umcutrecht.nl.

DOI:10.1523/JNEUROSCI.3280-07.2007

Copyright $\odot 2007$ Society for Neuroscience $\quad 0270-6474 / 07 / 2714139-08 \$ 15.00 / 0$
}

short time of action of NPY, which does not allow determination of whether these subtle differences in NPY signaling in the long term will contribute to obesity. Moreover, when infused in the PVN, NPY only elicits an effect when given in the early light period (van Dijk and Strubbe, 2003). Thus, in addition to differences in local effects of NPY, diurnal influences may also affect NPY responsiveness. Together, this raises the question how NPY influences diurnal feeding patterns, meal frequency and size when continuously infused in the PVN or in the LH and whether this contributes to the development of obesity.

A similar distinct effect of acute NPY as observed for feeding behavior, exists for the endocrine and autonomic effects. NPY has clear endocrine and autonomic effects when infused in the PVN but not in the LH (Billington et al., 1994; Currie and Coscina, 1995). This raises the question whether long-term infusion of NPY in the PVN and LH has differential effects on body weight regulation and energy metabolism when infused for a longer period of time.

To address these questions, we used viral gene transfer to specifically deliver NPY in these two brain areas. Recombinant adeno-associated viral (rAAV) particles containing NPY cDNA were injected to induce local expression of NPY in the PVN or the $\mathrm{LH}$, to release NPY in an ectopic and paracrine manner, which is still present several weeks after injection. Rather than overexpressing NPY in the Arc, being the major source of NPY released 
in the PVN and LH, we injected AAV-NPY directly into the target area. A feature of AAV2 transduction in the brain is that the vector remains confined to the injection site (Passini et al., 2004; Sin et al., 2005). Indeed, our previous findings, using a similar strategy to overexpress peptide, showed that immunostaining was limited to the injected area, and not observed in projection areas (Kas et al., 2004; Tiesjema et al., 2007).

\section{Materials and Methods}

Animals. Male Wistar rats weighing 220-250 g were purchased from Charles River (Crl-Wu, Germany). They were individually housed in filtertop cages with ad libitum access to food (CRM pellets; Special Diet Services, Whitham, Essex, UK) and water. Animals were kept in a temperature- and humidity-controlled room $\left(21 \pm 2^{\circ} \mathrm{C}\right)$ under a $12 \mathrm{~h} \mathrm{light} /$ dark cycle (lights on at 7:00 A.M.). All experimental procedures were approved by the Committee for Animal Experimentation of the University of Utrecht (Utrecht, The Netherlands).

Surgical procedures. Rats were anesthetized with $0.1 \mathrm{ml} / 100 \mathrm{~g}$ hypnorm (Janssen Pharmaceutica, Beerse, Belgium) and $0.05 \mathrm{ml} / 100 \mathrm{~g}$ (intraperitoneally) dormicum (HoffmanLaRoche, Mijdrecht, The Netherlands). Transmitters (TA10TA-F40; Data Science International, St. Paul, MN) were placed in the abdominal cavity. Rats were left to recover for 3 weeks.

Seven days after baseline recordings, rats were anesthetized again as described above. Using a stereotax, rAAV-neuron-specific enolase (NSE)-NPY ( $n=12$; LH-NPY) or rAAV-NSEempty (used as control) ( $n=7$; LH-contr) was injected bilaterally into the $\mathrm{LH}$ [coordinates: anteroposterior (AP), $-3.0 \mathrm{~mm}$ from bregma; mediolateral (ML), $\pm 1.6 \mathrm{~mm}$ from bregma; dorsoventral (DV), $-8.6 \mathrm{~mm}$ below the skull]. In a second experiment, rats were injected bilaterally into the PVN [coordinates: AP, $-1.8 \mathrm{~mm}$ from bregma; ML, $\pm 0.3 \mathrm{~mm}$ from bregma; $\mathrm{DV},-8.1$ $\mathrm{mm}$ below the skull; rAAV-NSE-NPY, $n=9$ (PVN-NPY); rAAV-NSE-empty, $n=7$ (PVNcontr)]. rAAV-NPY and rAAV-empty were a kind gift from M. J. During (University of Auckland, Auckland, New Zealand). Production of rAAV-NPY has been described previously (During et al., 2003; Richichi et al., 2004). Per site, 1 $\mu \mathrm{l}$ of virus $\left(1 \times 10^{8}\right.$ genomic copies $)$ was injected over $5 \mathrm{~min}$, after which the needle was kept in place for $10 \mathrm{~min}$ before removal. After each surgery, rats received an injection with 5 $\mathrm{mg} / \mathrm{kg}$ carprofen (Vericore, Dundee, UK).

Data analysis. One week before, until $50 \mathrm{~d}$ after viral injections, body weight, food intake, core temperature, and locomotor activity were recorded

Body weight gain and food intake were measured daily at 11:00 A.M. Core temperature and locomotor activity were automatically recorded via the transmitters that send digitized data via radio frequency signals to a nearby receiver. The data were recorded every $10 \mathrm{~min}$ using DSI software (DSI, St. Paul, MN). On days 21 and 48, meal patterns were recorded. Food hoppers were weighed automatically and data were sent to a computer every $12 \mathrm{~s}$ for $24 \mathrm{~h}$. A meal was defined as an episode of food (B) or LH $(\boldsymbol{D})$.
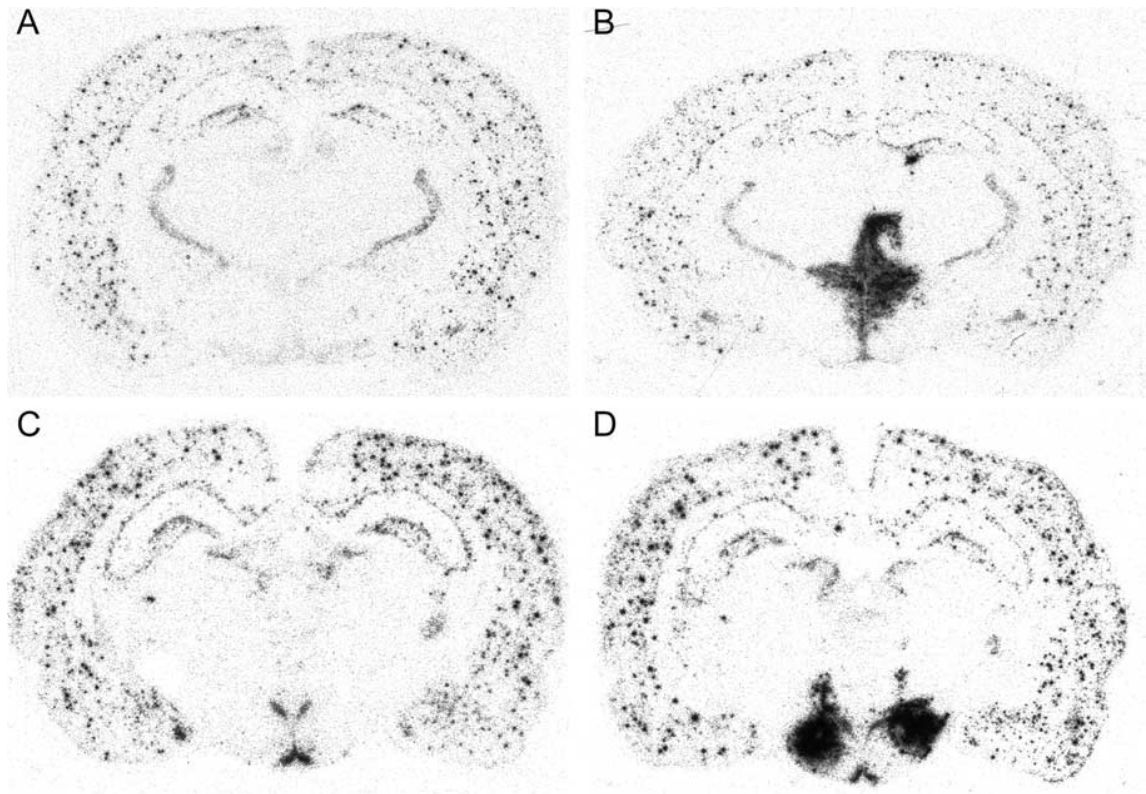

Figure 1. $\boldsymbol{A}-\boldsymbol{D}, \mathrm{NPY}$ mRNA expression in rats injected with rAAV-empty in the PVN $(\boldsymbol{A})$ or LH $(\boldsymbol{C})$, or with rAAV-NPY in the PVN
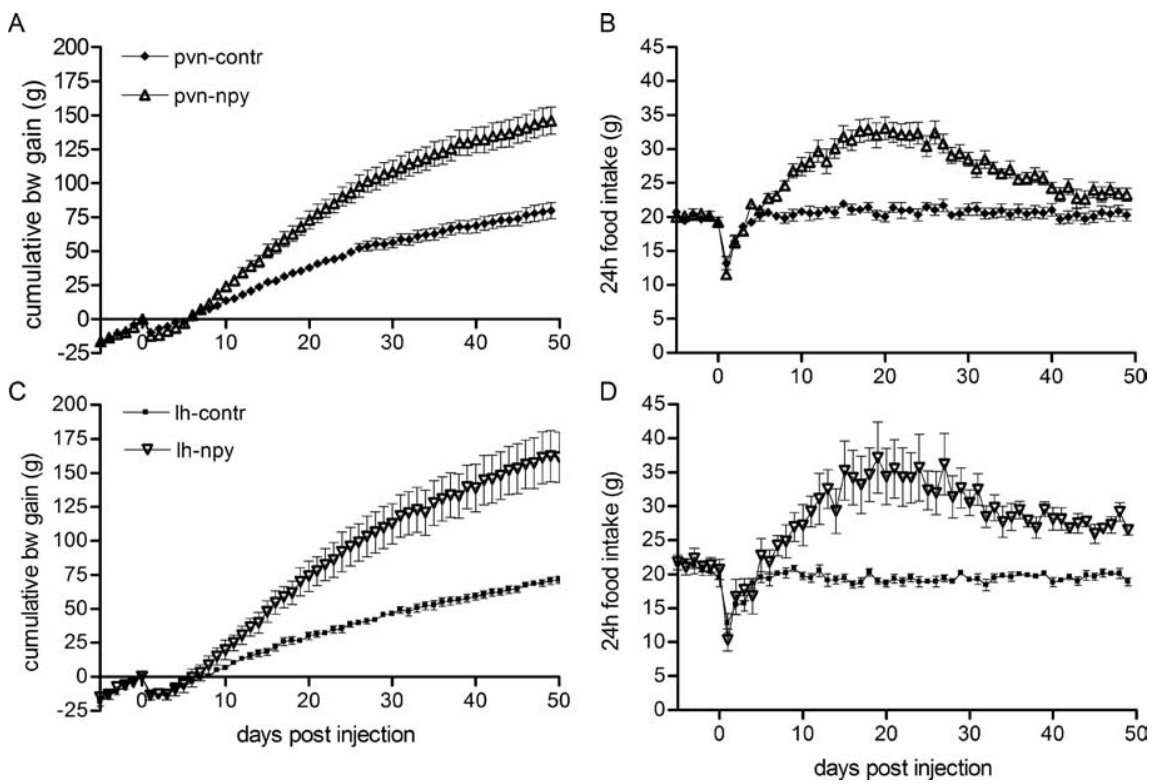

Figure 2. $\boldsymbol{A}-\boldsymbol{D}$, rAAV-NPY injections in the PVN or LH increase body weight $(\boldsymbol{A}, \boldsymbol{C})$ and food intake $(\boldsymbol{B}, \boldsymbol{D})$ compared with rAAV-contr rats. In PVN-NPY-injected rats, food intake declines to control levels $(\boldsymbol{B})$, whereas in LH-NPY-injected rats food intake is increased over the total time measured $(\boldsymbol{D})$. Data are represented as mean $\pm \operatorname{SEM}(n=5-6)$.

intake with a minimal consumption of $0.5 \mathrm{~g}$ of chow, and an intermeal interval of $5 \mathrm{~min}$.

Collection of blood and tissues. At day 50 after injection of the virus, rats were decapitated in the morning, and trunk blood was collected in heparinized tubes, after adding $83 \mu \mathrm{mol}$ of EDTA and $1 \mathrm{mg}$ aprotinin, and was immediately placed on ice. Plasma samples were stored at $-20^{\circ} \mathrm{C}$ until additional analysis.

Brains were immediately removed after decapitation, quickly frozen in cold isopentane $\left(-35^{\circ} \mathrm{C}\right)$, and stored at $-80^{\circ} \mathrm{C}$. Retroperitoneal, epididymal, mesenteric, and subcutaneous white adipose tissue was isolated and weighed. Pituitaries, adrenals, and the thymus were also isolated and weighed.

Quantitative in situ hybridization. Coronal sections of $16 \mu \mathrm{m}$ were used for radioactive in situ hybridization (raISH). ${ }^{33} \mathrm{P}$-labeled antisense 
RNA probes were made for agouti-related peptide (AgRP), NPY, proopiomelanocortin (POMC), melanin-concentrating hormone ( $\mathrm{MCH})$, and prepro-orexin. raISH procedure has been described previously (Hillebrand et al., 2006). Expression of AgRP, NPY, and POMC in the arcuate nucleus and expression of $\mathrm{MCH}$ and prepro-orexin in the $\mathrm{LH}$ were quantified using Image J software (National Institutes of Health, Bethesda, MD). Viral infection was localized by in situ hybridization with a digoxigenin-labeled woodchuck posttranscriptional regulatory element (WPRE) probe. The WPRE sequence is part of the expression cassette of all vectors used here. This ISH method has been described previously (Smits et al., 2005). Expression of NPY was also measured in the PVN and LH to further confirm viral-mediated overexpression.

Plasma analysis. Plasma leptin, insulin, and corticosterone were analyzed in duplicate using radioimmunoassay kits [Linco Research (St. Charles, MO) for leptin and insulin; ICN Biochemicals (Costa Mesa, CA) for corticosterone]. Plasma adrenocorticotropic hormone (ACTH) was measured in duplicate using a specific rabbit antiserum directed to the midportion of ACTH, which was kindly provided by Dr. G. B. Makara (Budapest, Hungary). Synthetic human $\mathrm{ACTH}_{(1-39)}$ (Peninsula Laboratories, Belmont, CA) was labeled with ${ }^{125} \mathrm{I}$ and used as tracer (Nijsen et al., 2000). Plasma glucose was measured in triplicate using a glucose/ GOD-Perid method (Boehringer Mannheim, Mannheim, Germany).

Statistical analysis. Data are presented as group means \pm SEM. Differences in body weight and food intake were assessed using repeatedmeasure analysis. When significant overall interactions were found, post $h o c$ analyses were performed with $t$ tests. Additional statistical analysis was performed with $t$ tests and correcting for multiple testing (Bonferroni). Differences were being considered significant at $p<0.05$.

\section{Results}

\section{Verification of AAV injections}

Correct injection of viral particles was verified by radioactive WPRE in situ hybridization. Rats were only included in the study when WPRE was observed bilaterally in the PVN (inclusion criteria: AP, between -1.3 and $2.3 \mathrm{~mm}$ from bregma; ML, between 0 and $1 \mathrm{~mm}$ from bregma) or LH (inclusion criteria: AP, between -2.3 and $3.8 \mathrm{~mm}$ from bregma; ML, between 1 and $2 \mathrm{~mm}$ from bregma). Animals with incorrect injections were excluded from analysis (in total, two rats from PVN-contr, three rats from PVNNPY, one rat from LH-contr, and seven rats from LH-NPY were excluded because of unilateral NPY expression or expression not restricted to the PVN or LH). Viral-induced NPY expression in the LH and PVN was confirmed by NPY in situ hybridization (Fig. 1). Previously, we showed that this increase in NPY mRNA also results in increased NPY peptide (Tiesjema et al., 2007).

\section{AAV-NPY-induced effects on body weight and food intake}

The first days after injection of the viral particles, all groups of rats showed a similar decrease in body weight gain and intake of food and water. All animals were on presurgery body weight within 1 week after injection. Both PVN-NPY rats and LH-NPY rats showed a significant increase in body weight gain from day 14 and day 21 after injection, respectively, when compared with controls (Fig. $2 \mathrm{~A}, \mathrm{C}$ ). In the last $10 \mathrm{~d}$ of the study, body weight gain of PVN-NPY rats was not significantly different from PVNcontrols; however, body weight gain of LH-NPY rats remained increased until the end of the study. At day 50, PVN-NPY rats accumulated $67 \%( \pm 11.5)$ more body weight compared with PVN-contr rats, whereas LH-NPY rats accumulated $127 \%$ ( \pm 25.7 ) more body weight compared with LH-contr rats.

Daily food intake of PVN-NPY-injected rats was significantly increased from day 8 after injection until day $40(p<0.001)$. Between day 40 and day 50, food intake was not significantly different between the PVN-NPY-injected group and the controls (Fig. 2 B). Food intake corrected for body weight was significantly
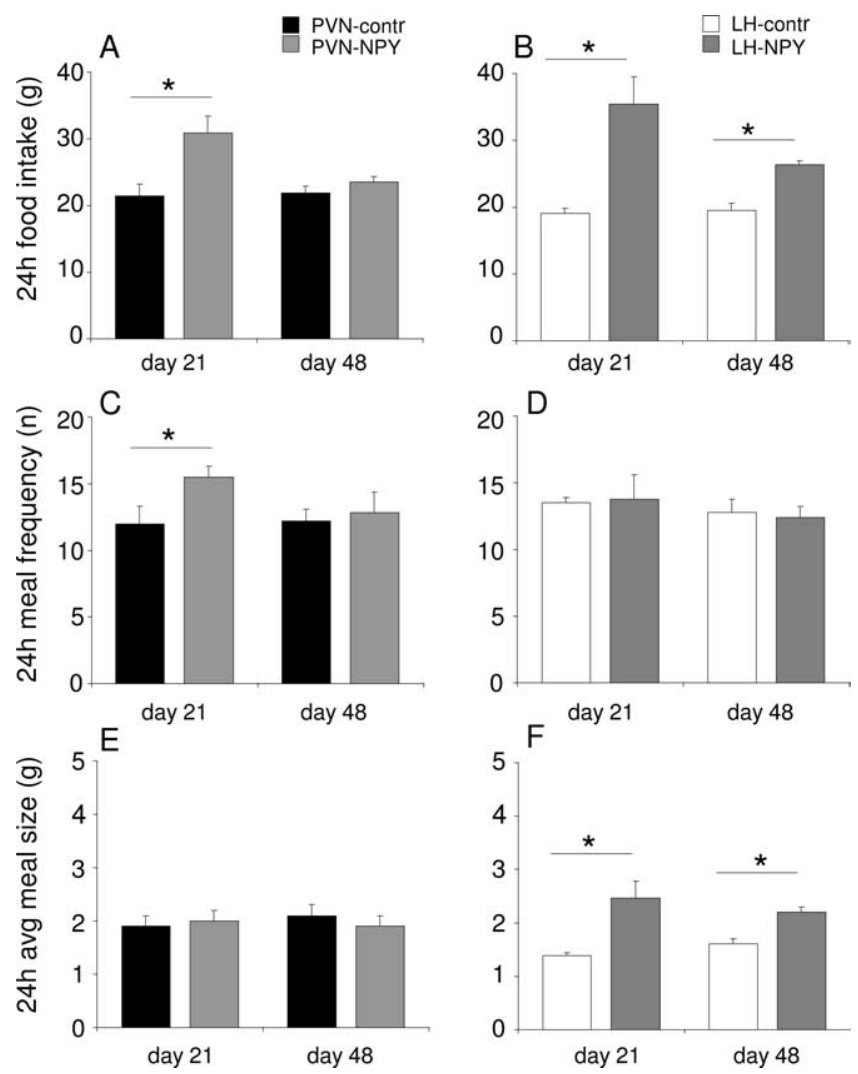

Figure 3. $\boldsymbol{A}, \boldsymbol{B}$, Food intake is increased on day 21 in rats with rAAV-NPY injections in the PVN $(\boldsymbol{A})$ or in the $\mathrm{LH}(\boldsymbol{B})$ compared with controls, whereas on day 48 food intake is only increased in LH-NPY-injected rats compared with controls $(\boldsymbol{B}) . \boldsymbol{C}, \boldsymbol{E}$, In the PVN-NPY-injected rats, increased food intake is attributable to increased meal frequency $(\boldsymbol{C})$ and not meal size $(\boldsymbol{E}) . \boldsymbol{D}, \boldsymbol{F}$, In the LH-NPY-injected rats, increased food intake is attributable to an increase in meal size $(\boldsymbol{F})$ and not meal frequency $(\boldsymbol{D})$. Data are represented as mean \pm SEM $(n=5-6) .{ }^{*} p<0.05 ;{ }^{* *} p<$ 0.01

increased from day 15 until day 35; after that, food intake corrected for body weight was similar between the two groups (day 40: $5.49 \pm 0.14 \mathrm{~g} / 100 \mathrm{~g}$ body weight vs $5.32 \pm 0.11 \mathrm{~g} / 100 \mathrm{~g}$ body weight for PVN-contr vs PVN-NPY). In LH-NPY rats, food intake was significantly increased from day 11 until the end of the experiment $(p<0.001)$ (Fig. $2 D)$. In contrast to PVN-NPYinjected rats, LH-NPY-injected rats still showed increased food intake when corrected for body weight at the end of the study (day 49: $5.32 \pm 0.16 \mathrm{~g} / 100 \mathrm{~g}$ body weight vs $4.79 \pm 0.16 \mathrm{~g} / 100 \mathrm{~g}$ body weight for LH-NPY vs LH-contr; $p<0.05$ ).

Daily water intake of PVN-NPY rats followed the food intake pattern, and was significantly increased between days 11 and 29 when compared with PVN-contr rats (average of $28.2 \pm 1.37$ vs $23.1 \pm 1.10 \mathrm{ml} / \mathrm{d}$ for PVN-NPY vs PVN-contr; $p<0.05$ ). There were no differences observed in water intake of LH-NPY and LH-contr rats (data not shown).

\section{AAV-NPY-induced effects on meal pattern}

At day 21, PVN-NPY rats showed an increase in food intake (Fig. $3 A$ ) because of an increase in light phase food intake (Table 1). Although $24 \mathrm{~h}$ food intake at day 48 was not significantly increased anymore in PVN-NPY rats (Fig. $3 A$ ), the food intake in the light period was slightly but significantly increased (Table 1). In LH-NPY rats, $24 \mathrm{~h}$ food intake was increased on days 21 and 48 (Fig. 3B). On day 21, this increase was attributable to an increase in food intake in both the light and dark phase within the LH- 
NPY group of rats; the amount of food consumed in the light phase was not significantly different from that consumed in the dark phase (Table 1). On day 48, the increase in food intake in LH-NPY rats was only attributable to an increase in light phase feeding, thus losing the diurnal rhythm of food intake (Table 1). Thus, in PVN-NPY and in LH-NPY rats, food intake is increased at $21 \mathrm{~d}$ and only in LHNPY rats we observed increased food intake at $48 \mathrm{~d}$ with a loss of the difference between the amount consumed in the dark and light period.

We next determined whether the effects of NPY on food intake were attributable to effects on meal size or meal frequency. At day 21, PVN-NPY rats showed an increased meal frequency compared with PVN-contr rats (Fig. $3 C$ ). This increase was attributable to more meals consumed in the light phase (Fig. 4A). The latency of the first meal was significantly decreased compared with control animals (PVN-contr, $441 \mathrm{~min} \pm 62.3$, vs PVN-NPY, $300 \mathrm{~min} \pm 29.4$; $p<0.03$ ). At day 48 , meal frequency was similar compared with controls (Figs. 3C, 4B). Meal size in PVN-NPY was not significantly different at either time point compared with controls (Figs. 3E, 4D).

At day 21, meal frequency in LH-PVN rats was not significantly different over $24 \mathrm{~h}$ compared with controls (Figs. 3D, 4E). Also, at day $48,24 \mathrm{~h}$ meal frequency was not significantly different (Fig. 3D); however, LH-NPY rats consumed as many meals in the light as in the dark phase (Fig. $4 F$ ). The latency to the first meal was not different between LH-NPY rats and LH-contr rats (data not shown). Meal size in LH-NPY rats was increased in both the dark and light phase of days 21 and 48 compared with controls (Figs. 3F, 4G,H).

Thus, in PVN-NPY rats, food intake is increased because of increased meal frequency, whereas in the LH-NPY rats, food intake is increased by increasing meal size. Although on day 48, LH-NPY rats increased meal frequency in the light phase, they compensated this increase in the dark phase by decreasing the numbers of meals taken.

\section{AAV-NPY-induced effects on core temperature and locomotor activity}

Dark phase core temperature was significantly decreased in both PVN-NPY and LH-NPY rats when compared with controls at days 21 and 48 (Table 2). In contrast, both groups of rats injected with $\mathrm{rAAV}$-NPY showed a significant increase in core temperature in the light phase of day 21 . On day 48 , however, no differences were observed in light phase core temperature (Table 2).

We further analyzed the daily rhythm of core temperature (Fig. 5, Table 3). The opposite changes in dark and light phase core temperature on day 21 resulted in a normal mean $24 \mathrm{~h}$ temperature in both PVN-NPY and LH-NPY rats. On day 48, however, both PVN-NPY and LH-NPY rats had a reduced mean $24 \mathrm{~h}$ temperature. Although a significant rhythm in temperature could be found in most animals injected with AAV-NPY, the amplitude of this rhythm was significantly reduced on both time points measured. Furthermore, the acrophase (peak time) was significantly advanced in PVN-NPY rats.

Locomotor activity of rats injected with rAAV-NPY in the PVN was reduced only in the dark phase of days 21 and 48, whereas activity of LH-NPY rats was, in addition to in the dark phase of day 21 and 48, also reduced in the light phase of day 21 (Table 2).
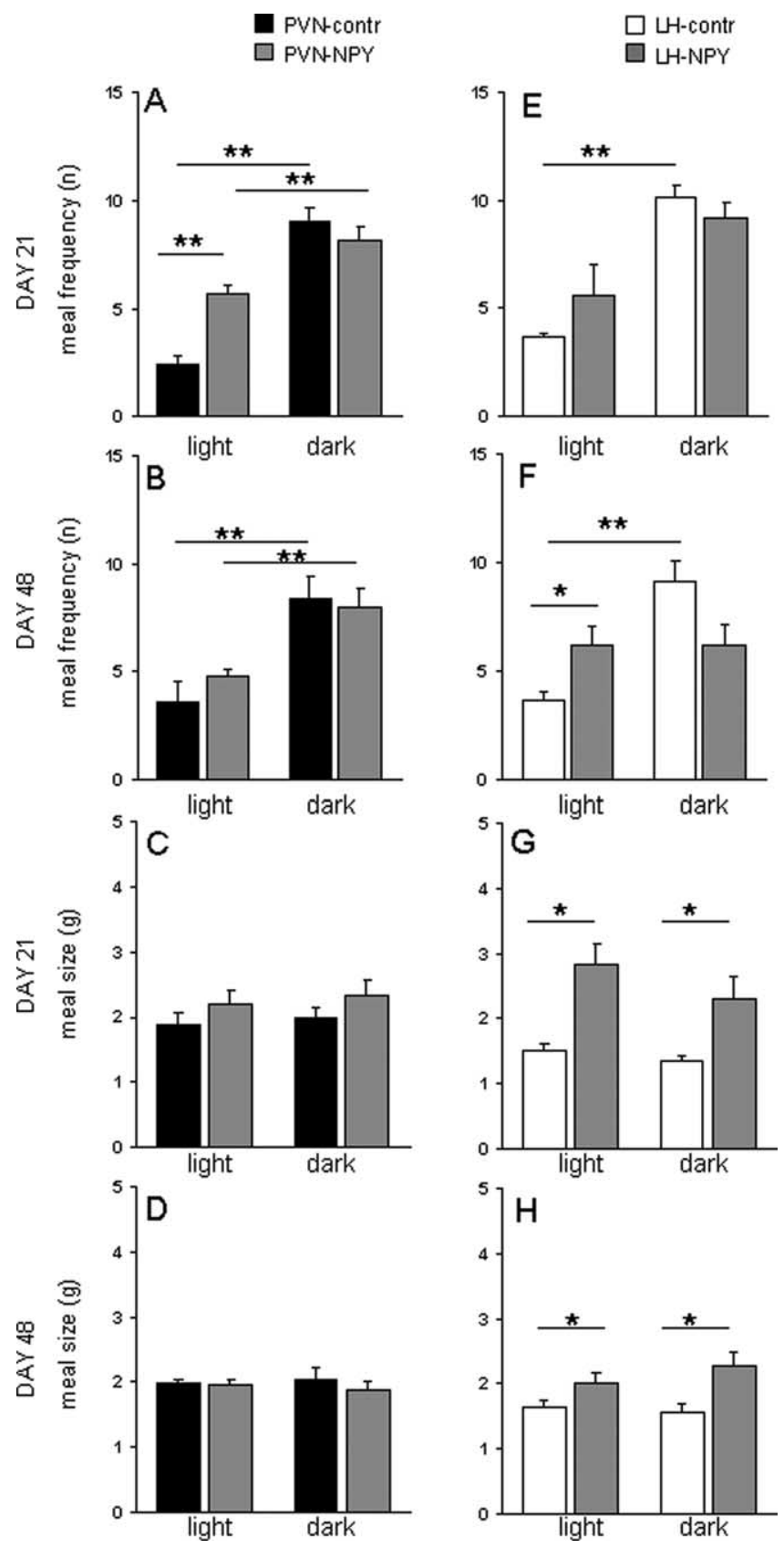

Figure 4. Differences in meal patterns over the light/dark cycle in rats injected with rAAVNPY in the PVN or in the LH. $\boldsymbol{A}, \boldsymbol{B}, 0$ n day 21, meal frequency is increased in PVN-NPY rats only in the light phase, and not in the dark phase $(\boldsymbol{A})$, and no differences are observed on day $48(\boldsymbol{B}) . \boldsymbol{C}$, $\boldsymbol{D}$, Meal size is not different in PVN-NPY-injected rats compared with controls. $\boldsymbol{E}, \boldsymbol{F}$, On day 21 , in LH-NPY-injected rats, meal frequency is not different in dark or light phase $(\boldsymbol{E})$, whereas on day 48 , the frequency in the light phase is significantly increased and not different than the frequency in the dark phase $(\boldsymbol{F}), \boldsymbol{G}, \boldsymbol{H}$, At day $21(\boldsymbol{G})$ and day $48(\boldsymbol{H})$, meal size is increased in LH-NPY-injected rats compared with controls. Data are represented as mean \pm SEM $(n=$ 5-6). ${ }^{*} p<0.05 ;{ }^{* *} p<0.01$. 
Table 2. Effects of rAAV-NPY injections in the PVN or LH on average core temperature and locomotor activity

\begin{tabular}{|c|c|c|c|c|c|}
\hline & & PVN-contr & PVN-NPY & LH-contr & LH-NPY \\
\hline \multicolumn{6}{|c|}{ Body temperature $\left({ }^{\circ} \mathrm{C}\right)$} \\
\hline \multirow[t]{2}{*}{ Day 21} & Light & $36.92 \pm 0.057$ & $37.08 \pm 0.024^{*}$ & $36.99 \pm 0.041$ & $37.19 \pm 0.063^{*}$ \\
\hline & Dark & $37.78 \pm 0.039$ & $37.50 \pm 0.036^{* *}$ & $37.67 \pm 0.031$ & $37.47 \pm 0.079^{*}$ \\
\hline \multirow[t]{2}{*}{ Day 48} & Light & $36.89 \pm 0.042$ & $36.89 \pm 0.042$ & $36.98 \pm 0.072$ & $37.03 \pm 0.056$ \\
\hline & Dark & $37.64 \pm 0.060$ & $37.26 \pm 0.065^{* *}$ & $37.63 \pm 0.054$ & $37.25 \pm 0.132^{*}$ \\
\hline \multicolumn{6}{|c|}{ Activity (\% basal) } \\
\hline \multirow[t]{2}{*}{ Day 21} & Light & $94.6 \pm 8.7$ & $89.5 \pm 7.6$ & $87.7 \pm 4.7$ & $63.1 \pm 7.8^{*}$ \\
\hline & Dark & $116.9 \pm 9.2$ & $72.5 \pm 5.7^{* *}$ & $85.1 \pm 4.5$ & $55.4 \pm 9.1^{*}$ \\
\hline \multirow[t]{2}{*}{ Day 48} & Light & $75.6 \pm 11.3$ & $85.5 \pm 11.4$ & $87.5 \pm 6.9$ & $76.4 \pm 13.3$ \\
\hline & Dark & $102.5 \pm 11.4$ & $66.5 \pm 5.3^{* *}$ & $83.2 \pm 6.3$ & $48.8 \pm 8.5^{*}$ \\
\hline
\end{tabular}

Activity data are presented as percentage of values before injection.

${ }^{*} p<0.05,{ }^{* *} p<0.01$ compared with controls.
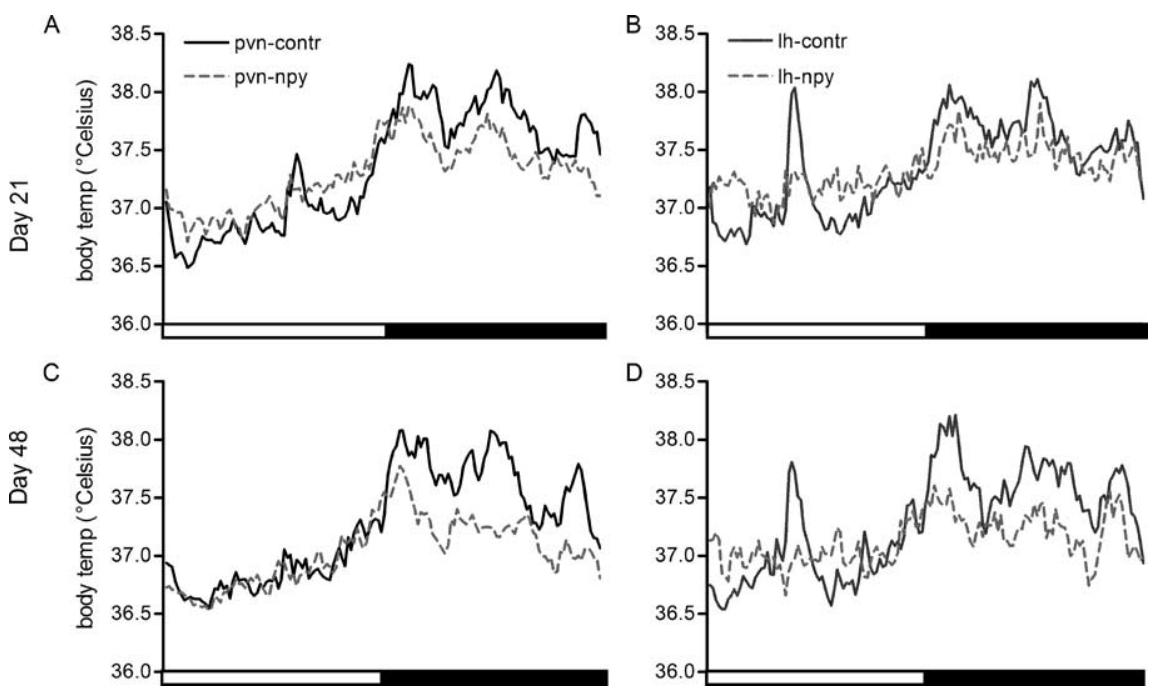

Figure 5. $A-D$, Effects of rAAV-NPY injections in the PVN or LH (data averaged per group) on daily temperature rhythm on day $21(\boldsymbol{A}, \boldsymbol{B})$ or day $48(\boldsymbol{C}, \boldsymbol{D})$. The light bar represents the $12 \mathrm{~h}$ light phase, and the black bar represents the $12 \mathrm{~h}$ dark phase. Data are represented as mean $(n=5-6)$. Average effects on core temperature and locomotor activity over light and dark phase are summarized in Table 2.

\section{AAV-NPY-induced effects on endocrine parameters and body composition}

Table 4 summarizes the effects of NPY overexpression in the PVN and LH on endocrine parameters and body composition.

Subcutaneous and abdominal fat pads and plasma concentrations of leptin and insulin were significantly increased in both PVN-NPY and LH-NPY rats. Whereas plasma glucose levels were normal in rats injected with rAAV-NPY in the $\mathrm{LH}$, rats injected in the PVN displayed increased concentrations of plasma glucose when compared with controls.

Plasma concentrations of both corticosterone and ACTH were increased in PVN-NPY rats when compared with PVNcontr rats, but not significantly different between LH-NPY and $\mathrm{LH}$-contr rats. No differences were found in the weight of the thymus or adrenal glands in both PVN-NPY and LH-NPY rats compared with controls.

\section{AAV-NPY-induced effects on neuropeptide expression in the Arc and LH}

Fifty days after injection of the viral particles, mRNA expression of NPY, AgRP, and POMC in the arcuate nucleus, and orexin and $\mathrm{MCH}$ in the LH was measured by raISH (Fig. 6). When compared with control rats, rats injected with rAAV-NPY in both the PVN and $\mathrm{LH}$ showed a similar reduction of AgRP mRNA in the Arc, but no change in POMC mRNA (Fig. $6 A, B)$. In LH-NPY, but not PVN-NPY rats, also expression of NPY mRNA was significantly reduced (Fig. 6C). Expression of orexin and $\mathrm{MCH}$ in the $\mathrm{LH}$ were not altered in rats injected with rAAV-NPY when compared with rats injected with rAAV-contr (Fig. 6C,D).

\section{Discussion}

Both in the PVN and in the LH, overexpression of NPY resulted in obesity with increased food intake, decreased darkphase core temperature, and decreased nonspecific locomotor activity. Interestingly, however, the increased food intake attributable to NPY overexpression in the PVN was explained by increased meal frequency, whereas in the LH, it was explained by increased meal size and changes in the diurnal pattern. A second clear difference between overexpression of NPY in the PVN and in the LH was observed in longterm feeding behavior. In the PVN, NPY overexpression only increased food intake temporarily for a few weeks, after which it decreased to levels observed in control animals. In contrast, in the LH, NPY significantly increased food intake (because of increased meal size) over the total period of $50 \mathrm{~d}$, despite a small decrease after 4 weeks. In addition, in LH-NPY rats, but not in PVN-NPY rats, diurnal rhythmicity with regard to food intake and body core temperature was lost. A comparison of effects of NPY overexpression in the PVN and LH is presented in Table 5.

Meal pattern analysis revealed that, in the period when daily food intake was maximal, PVN-NPY rats only increased food intake in the light phase by advancing the start of feeding by $2.5 \mathrm{~h}$. Interestingly, $\mathrm{NPY}^{-1-}$ mice show a delay in fasting-induced refeeding, an effect that is more pronounced when food is returned at the start of the light phase (Segal-Lieberman et al., 2003). A role for the PVN in this light-phase feeding, is also in agreement with the finding that NPY infused in the PVN affects food intake when injected in the light period and not when injected in the dark period (Tempel and Leibowitz, 1990; van Dijk and Strubbe, 2003). Moreover, in rats injected with AAV-NPY in the $\mathrm{LH}$, food intake was increased in both light and dark phase. This confirms earlier data by Stanley and Thomas (1993), who showed an increase in food intake after NPY infusion in the LH regardless of the time of day it was given. Here, we clearly show that there are distinct roles for NPY when expressed in LH or PVN in feeding behavior and the diurnal feeding pattern, and that this long-term increased NPY signaling is not compensated for and results in the development of obesity.

Increased feeding of PVN-NPY rats was attributable to an increase in the frequency of normal-sized meals, whereas LHNPY rats consumed larger meals. Thus, the location of overexpression affects how feeding behavior is modified. This is in agreement with previous findings in which NPY was locally infused in the PVN or LH (Kalra et al., 1988; Marin Bivens et al., 
1998), however contradict the findings of Leibowitz and Alexander (1991), who showed an effect of acute injections of NPY in the PVN on meal size. This discrepancy could be explained by the fact that our results are from long-term NPY overexpression, in contrast to rats in which NPY was injected only once. Indeed, a 4 h NPY infusion of NPY in the third ventricle increased only meal frequency (Kalra et al., 1988).

NPY overexpression in PVN resulted in temporal increase in feeding, whereas in $\mathrm{LH}$ it resulted in a chronic increase in feeding. It could be that, with NPY overexpression in the PVN, compensatory mechanisms are effective to decrease food intake to control levels, reflecting a new level of defended body weight. Interestingly, this does not occur in LH-injected rats. Therefore, we investigated whether the differences in PVN-NPY and LH-NPY rats were reflected by differences in expression level of NPY, AgRP, Orexin, MCH, and POMC mRNA. In both groups, a decrease in AgRP was observed, and in the LH rats, NPY overexpression also decreased NPY mRNA in the arcuate nucleus. Other measured neuropeptides were not changed. Thus, these changes do not explain the differences observed. A more extensive analysis of time-dependent changes (over the light/dark cycle as well as over days) is needed to understand underlying mechanisms.

One could argue that, because of feeding being increased, water intake should also be increased. Indeed, acute injections of NPY in the PVN result in an increase in water intake, although this is not observed after injections of NPY in the LH (Stanley et al., 1985). In line with this, we observed a small increase in water intake in PVN-NPY rats, whereas LH-NPY rats drank the same amount of water as controls, despite their increase in food intake. Thus, the increased drinking behavior of PVN-NPY rats is not simply a reflection of increased feeding behavior. This is strengthened by the fact that the drinking response is also observed in the absence of food when NPY is injected into the PVN (Stanley and Leibowitz, 1984).

Thus far, acute effects of NPY in the PVN on body temperature were contradictory (Currie and Coscina, 1995; Jolicoeur et al., 1995), and effects of NPY in the LH depend on the dose injected (Bouali et al., 1995; Jolicoeur et al., 1995). Moreover, it has been hypothesized previously that, although NPY signaling in the LH/PFA is important for energy intake, it is not involved in energy expenditure (i.e., temperature and respiratory quotient were unchanged after an acute injection in the PFA) (Bouali et al., 1995; Jolicoeur et al., 1995; Currie and Coscina, 1996). We show that long-term increased NPY signaling in both PVN and LH decreases core temperature. This change in core temperature was independent of feeding behavior, because feeding behavior was only temporally changed after PVN injection, whereas darkphase core temperature was decreased over the total $50 \mathrm{~d}$ of the experiment. Changes in core temperature may result from changes in diet-induced thermogenesis or from changes in heat loss mechanisms [for example, via sympathetic activity affecting tail blood flow (O’Leary et al., 1985)]. However, also nonspecific activity remained low after feeding behavior normalized in NPY-
A
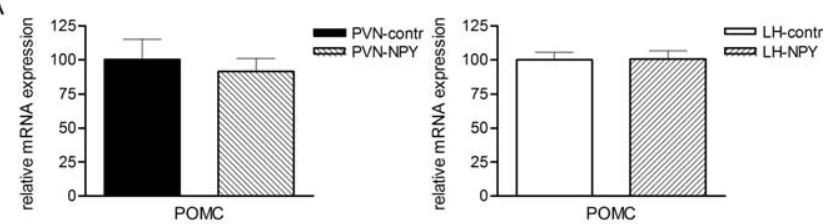

B
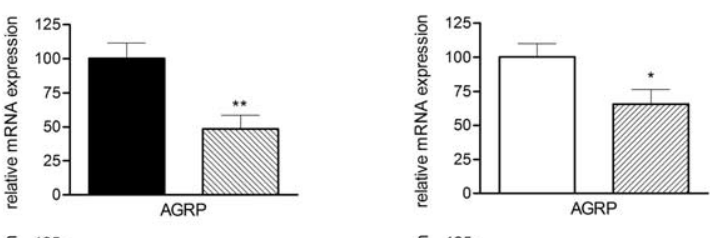

C

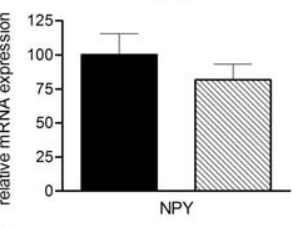

D
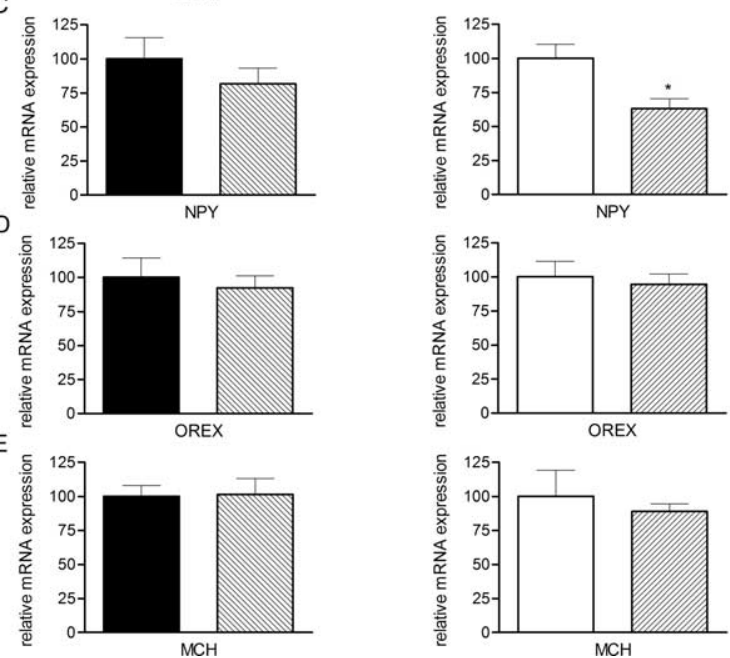

Figure 6. $\boldsymbol{A}-\boldsymbol{E}$, Effects of $\mathrm{rAAV}-\mathrm{NPY}$ injections in the PVN or LH on mRNA expression of POMC $(\boldsymbol{A}), \operatorname{AgRP}(\boldsymbol{B}), \operatorname{NPY}(\boldsymbol{C})$ in the $\operatorname{Arc}$, and orexin $(\boldsymbol{D})$ and $\mathrm{MCH}(\boldsymbol{E})$ in the LH. Data are represented as mean \pm SEM $(n=5-6)$. ${ }^{*} p<0.05 ;{ }^{* *} p<0.01$. 
Table 5. Comparison of the effects observed after injection of AAV-NPY in the PVN and LH

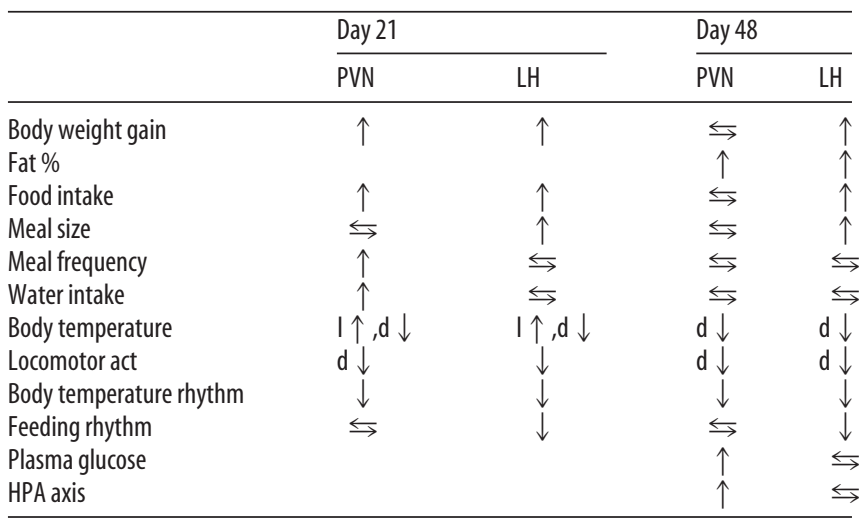

I, Light phase; d, dark phase.

PVN-injected rats, which could also have influenced core temperature. The precise mechanisms via which NPY affects core temperature and activity remain to be elucidated.

In addition to the effects on energy intake, core temperature, and nonspecific locomotor activity, we also uncovered a new role for increased NPY signaling in diurnal rhythmicity. PVN-NPY rats and LH-NPY rats display a reduced diurnal rhythmicity in core temperature, which is shown by a reduction of the overall amplitude. In PVN-NPY rats, this is accompanied by an advance in acrophase, probably reflecting the increase in light-phase feeding. Furthermore, despite the fact that PVN-NPY rats increased only their light-phase food intake, they still ingested more food during the dark phase than during the light phase, which is the normal time for a rat to eat. LH-NPY rats, however, consume as much food in the light phase as in the dark phase, probably because of induced wakefulness (Szentirmai and Krueger, 2006). Interestingly, the suprachiasmatic nucleus, which drives the diurnal rhythm of feeding activity, is hypothesized to accomplish this via projections to the $\mathrm{LH}$ (Abrahamson et al., 2001), but also direct projections from the suprachiasmatic nucleus to the arcuate nucleus are present (Yi et al., 2006). A continuous stimulation by increased NPY signaling in the LH would therefore explain the increased anticipation to eat as well as the time spent feeding. Together with the blunted temperature pattern of LH-NPY rats, this suggests a role for NPY in the LH in daily rhythms of both food intake and core temperature.

NPY is known to have a stimulating effect on the hypothalamic-pituitary-adrenal (HPA) axis (Zarjevski et al., 1993; Sainsbury et al., 1996), and in turn increased HPA axis activity is associated with obesity (Dallman et al., 2006). Therefore, we also investigated the effects of NPY overexpression on the HPA axis. Previously, we found that AAV-induced NPY overexpression in the PVN did not increase HPA axis activity $23 \mathrm{~d}$ after injection (Tiesjema et al., 2007). However, $50 \mathrm{~d}$ after injection, we observed an increase in plasma concentrations of ACTH and corticosterone in rats injected with AAV-NPY in the PVN. In contrast, rats injected with AAV-NPY in the LH did not alter the activity of the HPA axis. This suggests that the effects of NPY on the HPA axis are specifically regulated by the PVN, which is in line with the fact that corticotropin-releasing factor is produced in neurons of the PVN, but not in the LH (Hashimoto et al., 1982). Nevertheless, we showed that the effects on the HPA axis become evident at a later time point than the effects on food intake, indicating that these are independent effects of increased NPY signaling in the PVN.
In conclusion, long-term increased NPY signaling results in obesity by increasing food intake and decreasing nonspecific locomotor activity. For the effects of food intake, we clearly show region-specific effects. In the PVN, NPY expression affects the initiation of food intake and activation of the HPA axis, and in the LH, NPY expression increases meal size, and alters diurnal patterns, equalizing light- and dark-phase food intake and core temperature. This suggests that the NPY system differentially regulates energy intake and energy expenditure in a site-specific manner, which together adjust energy balance.

\section{References}

Abrahamson EE, Leak RK, Moore RY (2001) The suprachiasmatic nucleus projects to posterior hypothalamic arousal systems. NeuroReport 12:435-440.

Ammar AA, Sederholm F, Saito TR, Scheurink AJ, Johnson AE, Sodersten P (2000) NPY-leptin: opposing effects on appetitive and consummatory ingestive behavior and sexual behavior. Am J Physiol 278:R1627-R1633.

Baird JP, Gray NE, Fischer SG (2006) Effects of neuropeptide Y on feeding microstructure: dissociation of appetitive and consummatory actions. Behav Neurosci 120:937-951.

Benoit SC, Clegg DJ, Woods SC, Seeley RJ (2005) The role of previous exposure in the appetitive and consummatory effects of orexigenic neuropeptides. Peptides 26:751-757.

Billington CJ, Briggs JE, Harker S, Grace M, Levine AS (1994) Neuropeptide $\mathrm{Y}$ in hypothalamic paraventricular nucleus: a center coordinating energy metabolism. Am J Physiol 266:R1765-R1770.

Bouali SM, Fournier A, St Pierre S, Jolicoeur FB (1995) Effects of NPY and NPY2-36 on body temperature and food intake following administration into hypothalamic nuclei. Brain Res Bull 36:131-135.

Currie PJ, Coscina DV (1995) Dissociated feeding and hypothermic effects of neuropeptide $\mathrm{Y}$ in the paraventricular and perifornical hypothalamus. Peptides 16:599-604.

Currie PJ, Coscina DV (1996) Regional hypothalamic differences in neuropeptide $\mathrm{Y}$-induced feeding and energy substrate utilization. Brain Res 737:238-242.

Dallman MF, Pecoraro NC, La Fleur SE, Warne JP, Ginsberg AB, Akana SF, Laugero KC, Houshyar H, Strack AM, Bhatnagar S, Bell ME (2006) Glucocorticoids, chronic stress, and obesity. Prog Brain Res 153:75-105.

During MJ, Young D, Baer K, Lawlor P, Klugmann M (2003) Development and optimization of adeno-associated virus vector transfer into the central nervous system. Methods Mol Med 76:221-236.

Hashimoto K, Ohno N, Aoki Y, Kageyama J, Takahara J, Ofuji T (1982) Distribution and characterization of corticotropin-releasing factor and arginine vasopressin in rat hypothalamic nuclei. Neuroendocrinology 34:32-37.

Hillebrand JJ, Kas MJ, Scheurink AJ, van Dijk G, Adan RA (2006) AgRP(83132) and SHU9119 differently affect activity-based anorexia. Eur Neuropsychopharmacol 16:403-412.

Jolicoeur FB, Bouali SM, Fournier A, St Pierre S (1995) Mapping of hypothalamic sites involved in the effects of NPY on body temperature and food intake. Brain Res Bull 36:125-129.

Kalra SP, Dube MG, Kalra PS (1988) Continuous intraventricular infusion of neuropeptide $\mathrm{Y}$ evokes episodic food intake in satiated female rats: effects of adrenalectomy and cholecystokinin. Peptides 9:723-728.

Kas MJ, Tiesjema B, van Dijk G, Garner KM, Barsh GS, Brake OT, Verhaagen J, Adan RA (2004) Induction of brain-region-specific forms of obesity by agouti. J Neurosci 24:10176-10181.

Kowalski TJ, Farley C, Cohen-Williams ME, Varty G, Spar BD (2004) Melanin-concentrating hormone-1 receptor antagonism decreases feeding by reducing meal size. Eur J Pharmacol 497:41-47.

Leibowitz SF, Alexander JT (1991) Analysis of neuropeptide Y-induced feeding: dissociation of Y1 and Y2 receptor effects on natural meal patterns. Peptides 12:1251-1260.

Marin Bivens CL, Thomas WJ, Stanley BG (1998) Similar feeding patterns are induced by perifornical neuropeptide $\mathrm{Y}$ injection and by food deprivation. Brain Res 782:271-280.

Nijsen MJ, Croiset G, Stam R, Bruijnzeel A, Diamant M, de Wied D, Wiegant VM (2000) The role of the CRH type 1 receptor in autonomic responses to corticotropin-releasing hormone in the rat. Neuropsychopharmacology 22:388-399. 
O'Leary DS, Johnson JM, Taylor WF (1985) Mode of neural control mediating rat tail vasodilation during heating. J Appl Physiol 59:1533-1538.

Passini MA, Watson DJ, Wolfe JH (2004) Gene delivery to the mouse brain with adeno-associated virus. Methods Mol Biol 246:225-236.

Richichi C, Lin EJ, Stefanin D, Colella D, Ravizza T, Grignaschi G, Veglianese P, Sperk G, During MJ, Vezzani A (2004) Anticonvulsant and antiepileptogenic effects mediated by adeno-associated virus vector neuropeptide $\mathrm{Y}$ expression in the rat hippocampus. J Neurosci 24:3051-3059.

Sainsbury A, Rohner-Jeanrenaud F, Grouzmann E, Jeanrenaud B (1996) Acute intracerebroventricular administration of neuropeptide Y stimulates corticosterone output and feeding but not insulin output in normal rats. Neuroendocrinology 63:318-326.

Sederholm F, Ammar AA, Sodersten P (2002) Intake inhibition by NPY: role of appetitive ingestive behavior and aversion. Physiol Behav 75:567-575.

Seeley RJ, Payne CJ, Woods SC (1995) Neuropeptide Y fails to increase intraoral intake in rats. Am J Physiol 268:R423-R427.

Segal-Lieberman G, Trombly DJ, Juthani V, Wang X, Maratos-Flier E (2003) NPY ablation in C57BL/6 mice leads to mild obesity and to an impaired refeeding response to fasting. Am J Physiol 284:E1131-E1139.

Sin M, Walker PD, Bouhamdan M, Quinn JP, Bannon MJ (2005) Preferential expression of an AAV-2 construct in NOS-positive interneurons following intrastriatal injection. Brain Res Mol Brain Res 141:74-82.

Smits SM, Mathon DS, Burbach JP, Ramakers GM, Smidt MP (2005) Molecular and cellular alterations in the Pitx3-deficient midbrain dopaminergic system. Mol Cell Neurosci 30:352-363.

Stanley BG, Leibowitz SF (1984) Neuropeptide Y: stimulation of feeding and drinking by injection into the paraventricular nucleus. Life Sci 35:2635-2642.

Stanley BG, Thomas WJ (1993) Feeding responses to perifornical hypotha- lamic injection of neuropeptide $\mathrm{Y}$ in relation to circadian rhythms of eating behavior. Peptides 14:475-481.

Stanley BG, Chin AS, Leibowitz SF (1985) Feeding and drinking elicited by central injection of neuropeptide Y: evidence for a hypothalamic site(s) of action. Brain Res Bull 14:521-524.

Szentirmai E, Krueger JM (2006) Central administration of neuropeptide Y induces wakefulness in rats. Am J Physiol 291:R473-R480.

Tempel DL, Leibowitz SF (1990) Diurnal variations in the feeding responses to norepinephrine, neuropeptide $\mathrm{Y}$ and galanin in the PVN. Brain Res Bull 25:821-825.

Tiesjema B, La Fleur SE, Luijendijk MCM, Brans MAD, Lin EJ, During MJ, Adan RA (2007) Injection of rAAV-NPY in the paraventricular nucleus results in obesity. Obesity 15:2424-2435.

van Dijk G, Strubbe JH (2003) Time-dependent effects of neuropeptide Y infusion in the paraventricular hypothalamus on ingestive and associated behaviors in rats. Physiol Behav 79:575-580.

Williams DL, Grill HJ, Weiss SM, Baird JP, Kaplan JM (2002) Behavioral processes underlying the intake suppressive effects of melanocortin $3 / 4$ receptor activation in the rat. Psychopharmacology (Berl) 161:47-53.

Yi CX, van d V, Dai J, Yin G, Ru L, Buijs RM (2006) Ventromedial arcuate nucleus communicates peripheral metabolic information to the suprachiasmatic nucleus. Endocrinology 147:283-294.

Zarjevski N, Cusin I, Vettor R, Rohner-Jeanrenaud F, Jeanrenaud B (1993) Chronic intracerebroventricular neuropeptide-Y administration to normal rats mimics hormonal and metabolic changes of obesity. Endocrinology 133:1753-1758.

Zheng H, Patterson LM, Phifer CB, Berthoud HR (2005) Brain stem melanocortinergic modulation of meal size and identification of hypothalamic POMC projections. Am J Physiol 289:R247-R258. 